\title{
Front Matter: Volume 10807
}

, "Front Matter: Volume 10807," Proc. SPIE 10807, Photomask Japan 2018. XXV Symposium on Photomask and Next-Generation Lithography Mask Technology, 1080701 (20 July 2018); doi: 10.1117/12.2504156 


\title{
PROCEEDINGS OF SPIE
}

\section{Photomask Japan 2018: XXV Symposium on Photomask and Next-Generation Lithography Mask Technology}

\author{
Kiwamu Takehisa
}

Editor

18-20 April 2018

Yokohama, Japan

Organized by

Photomask Japan

SPIE

Co-organized by

BACUS

EMLC

In Cooperation with

The Japan Society of Applied Physics

The Japan Society for Precision Engineering

The Institute of Electrical Engineering (Japan)

Technical Exhibit in Cooperation with SEMI (Japan)

Supported by

City of Yokohama

Published by

SPIE 
The papers in this volume were part of the technical conference cited on the cover and title page. Papers were selected and subject to review by the editors and conference program committee. Some conference presentations may not be available for publication. Additional papers and presentation recordings may be available online in the SPIE Digital Library at SPIEDigitalLibrary.org.

The papers reflect the work and thoughts of the authors and are published herein as submitted. The publisher is not responsible for the validity of the information or for any outcomes resulting from reliance thereon.

Please use the following format to cite material from these proceedings:

Author(s), "Title of Paper," in Photomask Japan 2018: XXV Symposium on Photomask and NextGeneration Lithography Mask Technology, edited by Kiwamu Takehisa, Proceedings of SPIE Vol. 10807 (SPIE, Bellingham, WA, 2018) Seven-digit Article CID Number.

ISSN: 0277-786X

ISSN: 1996-756X (electronic)

ISBN: 9781510622012

ISBN: 9781510622029 (electronic)

Published by

SPIE

P.O. Box 10, Bellingham, Washington 98227-0010 USA

Telephone +1 3606763290 (Pacific Time) · Fax +1 3606471445

SPIE.org

Copyright (C) 2018, Society of Photo-Optical Instrumentation Engineers.

Copying of material in this book for internal or personal use, or for the internal or personal use of specific clients, beyond the fair use provisions granted by the U.S. Copyright Law is authorized by SPIE subject to payment of copying fees. The Transactional Reporting Service base fee for this volume is $\$ 18.00$ per article (or portion thereof), which should be paid directly to the Copyright Clearance Center (CCC), 222 Rosewood Drive, Danvers, MA 01923. Payment may also be made electronically through CCC Online at copyright.com. Other copying for republication, resale, advertising or promotion, or any form of systematic or multiple reproduction of any material in this book is prohibited except with permission in writing from the publisher. The CCC fee code is 0277$786 \mathrm{X} / 18 / \$ 18.00$.

Printed in the United States of America.

Publication of record for individual papers is online in the SPIE Digital Library.

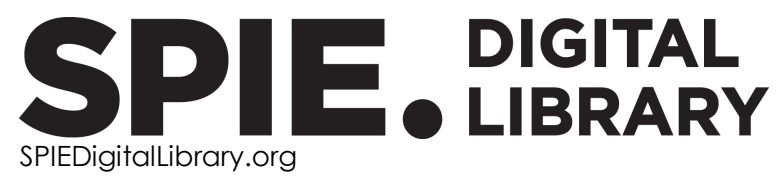

Paper Numbering: Proceedings of SPIE follow an e-First publication model. A unique citation identifier (CID) number is assigned to each article at the time of publication. Utilization of CIDs allows articles to be fully citable as soon as they are published online, and connects the same identifier to all online and print versions of the publication. SPIE uses a seven-digit CID article numbering system structured as follows:

$\square$ The first five digits correspond to the SPIE volume number.

- The last two digits indicate publication order within the volume using a Base 36 numbering system employing both numerals and letters. These two-number sets start with 00, 01, 02, 03, 04, 05, 06, 07, 08, 09, OA, OB ... 0Z, followed by 10-1Z, 20-2Z, etc. The CID Number appears on each page of the manuscript. 


\section{Contents}

$\begin{array}{ll}\vee & \text { Authors } \\ \text { vii } & \text { Conference Committee }\end{array}$

NIL

1080702 Progress in nanoimprint wafer and mask systems for high volume semiconductor manufacturing [10807-16]

1080704 Improved particle control for high volume semiconductor manufacturing for nanoimprint lithography [10807-18]

FPD PHOTOMASKS

1080705 Shin-Ełsu super-high-flat substrate for FPD panel photomask [10807-5]

$1080706 \quad$ New half-tone lithography uses for FPD proximity printing (Best Paper Award) [10807-7]

WRITING AND METROLOGY

$1080707 \quad$ Multi-beam mask writer MBM-1000 [10807-23]

PROCESS AND REPAIR

1080708 Dry etching technologies for Cr film [10807-22]

1080709 Update on the performance of photomask repair and clean with DUV femtosecond laser processes [10807-14]

EDA AND LITHOGRAPHY

10807 OA Demonstration of an effective mask proximity correction for advanced photomask [10807-19]

10807 OB Novel thermoplastic SOC materials for planarization use in multilayer lithography process [10807-10] 
10807 OC eBeam initiative surveys report greatly increased confidence in EUV and mask process correction requirements (Invited Paper) [10807-4]

10807 OD Minimizing "Tone Reversal" during 19x nm mask inspection (Best Paper Award) [10807-21]

10807 OE $\quad$ EUV capping layer integrity [10807-8]

10807 OF Fabrication of Ta based absorber EUV mask with SRAF [10807-25]

\section{EUV MASKS II}

$10807 \mathrm{OH} \quad$ Blank defect coverage budget for 16nm half-pitch single EUV exposure [10807-24]

EUV MASKS IV

10807 Ol Development of closed-type EUV pellicle (Best Paper Award) [10807-13]

POSTER SESSION: MASK TECHNOLOGIES

10807 OJ Advanced ceramic protective lifetime prolong for particle control [10807-2]

10807 OK Intra-field mask-to-mask overlay, separating the mask writing from the dynamic pellicle contribution (Best Poster Award) [10807-17]

10807 OL Achieve high hotspot detection accuracy by pattern scoring [10807-3]

10807 OM Enabling accurate and cost-effective registration metrology on EUVL masks [10807-15]

10807 ON EUV mask with advanced hybrid black border suppressing EUV and DUV OOB light reflection [10807-20]

\section{POSTER SESSION: MASK/LITHOGRAPHY RELATED TECHNOLOGIES IN ACADEMIA}

1080700 Exposure characteristics of ternary copolymerization positive tone electron beam resist containing $p$-chloro-a-methylstyrene [10807-12] 


\section{Authors}

Numbers in the index correspond to the last two digits of the seven-digit citation identifier (CID) article numbering system used in Proceedings of SPIE. The first five digits reflect the volume number. Base 36 numbering is employed for the last two digits and indicates the order of articles within the volume. Numbers start with 00, 01, 02, 03, 04, 05, 06, 07, 08, 09, 0A, 0B...0Z, followed by 10-1Z, 20-2Z, etc.

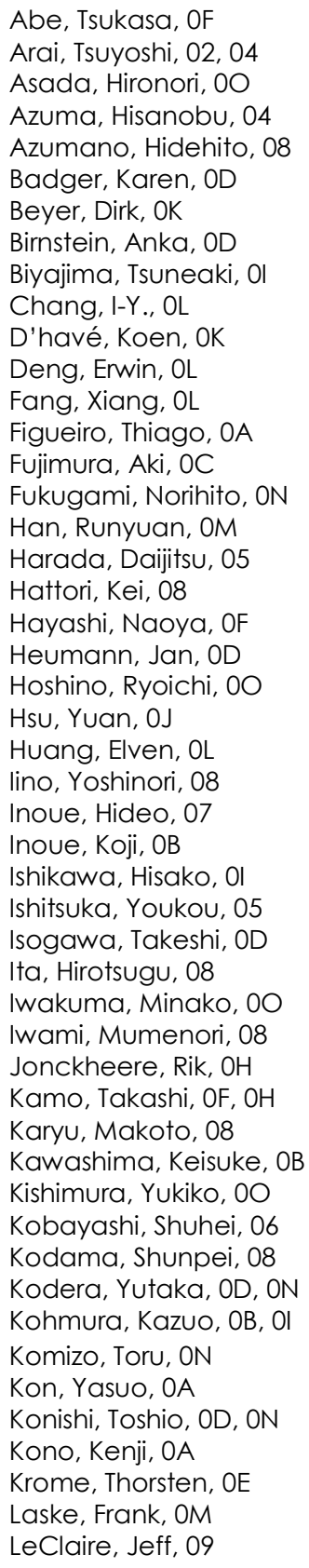

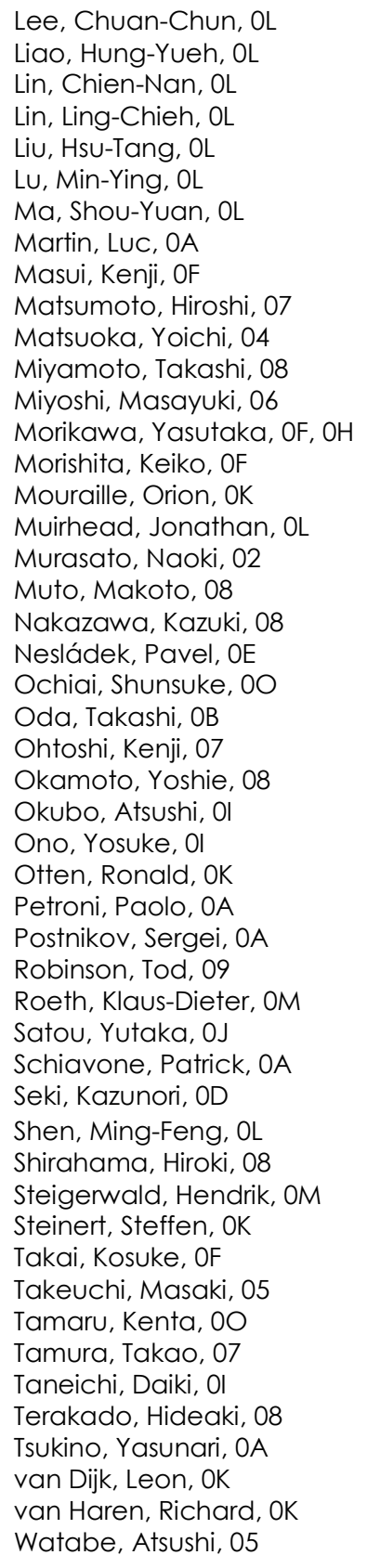


Watanabe, Genta, ON

Willis, Jan, $0 \mathrm{C}$

Yamane, Takeshi, $\mathrm{OH}$

Yamashita, Hiroshi, 07

Yang, Chuen-Huei, OL

Yonetani, Masashi, OD

Yoshida, Koichiro, 06

Yoshikawa, Yutaka, 06

Yoshimori, Tomoaki, 08

Proc. of SPIE Vol. 10807 1080701-6

Downloaded From: https://www.spiedigitallibrary.org/conference-proceedings-of-spie on 26 Apr 2023 Terms of Use: https://www.spiedigitallibrary.org/terms-of-use 


\title{
Conference Committee
}

\author{
Symposium Chair
}

Masato Shibuya, Tokyo Polytechnic University (Japan)

Symposium Vice Chair

Takeo Watanabe, University of Hyogo (Japan)

Advisory Committee Chair

Tadahiro Takigawa, ALITECS Corporation (Japan)

Advisory Committee

Naoya Hayashi, Dai Nippon Printing Company, Ltd. (Japan)

Morihisa Hoga, Consultant (Japan)

Toshiyuki Horiuchi, Tokyo Denki University (Japan)

Masanori Komuro, Consultant (Japan)

Masao Otaki, Consultant (Japan)

Yoshio Tanaka, D2S, Inc. (USA) and D2S K.K. (Japan)

Organizing Committee Chair

Masato Shibuya, Tokyo Polytechnic University (Japan)

Organizing Committee Vice Chair

Takeo Watanabe, University of Hyogo (Japan)

Organizing Committee

Uwe Behringer, UBC Microelectronics (Germany)

Junko Collins, SEMI Japan (Japan)

Brian J. Grenon, RAVE, LLC (USA)

Hideaki Hamada, HTL Company Japan Ltd. (Japan)

Eiichi Hoshino, Nikon Corporation (Japan)

Toshio Konishi, Toppan Printing Company, Ltd. (Japan)

Koji Mikami, Canon Inc. (Japan)

Hideaki Mitsui, HOYA Corporation (Japan)

Hiroyuki Miyashita, Dai Nippon Printing Company, Ltd. (Japan)

Junji Miyazaki, ASML Japan Company, Ltd. (Japan)

Warren Montgomery, Irresistible Materials (USA)

Hiroaki Morimoto, Toppan Printing Company, Ltd. (Japan) 
Kiwamu Takehisa, Lasertec Corporation (Japan)

Anto Yasaka, Hitachi High-Tech Science Corporation (Japan)

Auditors

Ichiro Kagami, Sony Semiconductor Manufacturing Corporation (Japan)

Yoji Tonooka, Toppan Printing Company, Ltd. (Japan)

Steering Committee Chair

Toshio Konishi, Toppan Printing Company, Ltd. (Japan)

Steering Committee Vice Chairs

Takashi Kamo, Toshiba Memory Corporation (Japan)

Kazuyuki Sukou, Dai Nippon Printing Company, Ltd. (Japan)

Steering Committee

Akihiko Ando, Toshiba Memory Corporation (Japan)

Junji Hirumi, Mie FUJITSU Semiconductor Ltd. (Japan)

Kunihiro Hosono, Renesas System Design Company, Ltd. (Japan)

Kokoro Kato, Nihon Synopsys G.K. (Japan)

Yutaka Kodera, Toppan Printing Company, Ltd. (Japan)

Yasutaka Morikawa, Dai Nippon Printing Company, Ltd. (Japan)

Naoki Nishida, HOYA Corporation (Japan)

Teruaki Noguchi, JEOL Ltd. (Japan)

Kiwamu Takehisa, Lasertec Corporation (Japan)

Nobuyuki Yoshioka, Dai Nippon Printing Company, Ltd. (Japan)

Shusuke Yoshitake, NuFlare Technology, Inc. (Japan)

Program Committee Chair

Kiwamu Takehisa, Lasertec Corporation (Japan)

Program Committee Vice Chairs

Akihiko Ando, Toshiba Memory Corporation (Japan)

Yutaka Kodera, Toppan Printing Company, Ltd. (Japan)

Program Committee

Tsukasa Abe, Dai Nippon Printing Company, Ltd. (Japan)

Hajime Aoyama, Nikon Corporation (Japan)

Peter Buck, Mentor Graphics Corporation (USA)

Thomas Faure, GLOBALFOUNDRIES Corporation (USA)

Kazuyuki Hagiwara, D2S K.K. (Japan)

Terumasa Hirano, HOYA Corporation (Japan) 
Hidemichi Imai, Dai Nippon Printing Company, Ltd. (Japan)

Franklin Kalk, Toppan Photomasks. Inc. (USA)

Takashi Kamikubo, NuFlare Technology, Inc. (Japan)

Izak Kapilevich, Applied Materials, Inc. (USA)

Bryan Kasprowicz, Photronics (USA)

Kokoro Kato, Nihon Synopsys G.K. (Japan)

Yusuke Kawano, Renesas Electronics Corporation (Japan)

Byung-Gook Kim, Samsung Electronics Company, Ltd. (Korea)

Masaaki Koyama, Sony Semiconductor Manufacturing Corporation

(Japan)

Yasuyuki Kushida, Mie FUJITSU Semiconductor Ltd. (Japan)

John Lin, Taiwan Semiconductor Manufacturing Company Ltd. (Taiwan)

Hironobu Manabe, JEOL Ltd. (Japan)

Junji Miyazaki, ASML Japan Company, Ltd. (Japan)

Yoshinori Nagaoka, KLA-Tencor Japan Ltd. (Japan)

Chiaki Sato, Canon Inc. (Japan)

Patrick Schiavone, ASELTA Nanographics (France)

Kazunori Seki, Toppan Printing Company, Ltd. (Japan)

Tsutomu Shoki, HOYA Corporation (Japan)

Yasunari Sohda, Hitachi, Ltd. (Japan)

Yasuko Tabata, TowerJazz Panasonic Semiconductor Company, Ltd. (Japan)

Nobuhiko Yabu, Canon Inc. (Japan)

Shingo Yoshikawa, Dai Nippon Printing Company, Ltd. (Japan)

Guojing Zhang, Intel Corporation (USA) 
Session Chairs

$1 \quad$ NIL

Akihiko Ando, Toshiba Memory Corporation (Japan)

Douglas Resnick, Canon Nanotechnologies, Inc. (USA)

2 FPD Photomasks

Nobuhiko Yabu, Canon Inc. (Japan)

Hajime Aoyama, Nikon Corporation (Japan)

3 Wring \& Metrology

Takashi Kamikubo, NuFlare Technology, Inc. (Japan)

Uwe Behringer, UBC Microelectronics (Germany)

4 Process \& Repair

Yasutaka Morikawa, Dai Nippon Printing Company, Ltd. (Japan)

5 EDA \& Lithography

Yoshinori Nagaoka, KLA-Tencor Japan Ltd. (Japan)

Bala Thumma, Synopsys, Inc. (USA)

6 EUV Masks (I)

Tsutomu Shoki, HOYA Corporation (Japan)

Jim Wiley, ASML (The Netherlands)

7 EUV Source for Inspection

Junji Miyazaki, ASML Japan (Japan)

Pavel Nesladek, Advanced Mask Technology Center GmbH \& Company KG (Germany)

$8 \quad 25^{\text {th }}$ Anniversary Special Session

Kiwamu Takehisa, Lasertec Corporation (Japan)

Kokoro Kato, Nihon Synopsys G.K. (Japan)

10 EUV Masks (II)

Yutaka Kodera, Toppan Printing Company, Ltd. (Japan)

Izak Kapilevich, Applied Materials, Inc. (USA)

11 EUV Masks (III)

Tsukasa Abe, Dai Nippon Printing Company, Ltd. (Japan)

Rik Jonckheere, Imec (Belgium)

12 EUV Masks (IV)

Kazunori Seki, Toppan Printing Company, Ltd. (Japan)

Jim Wiley, ASML (The Netherlands) 